\title{
Hemangioma y Linfangioma Mixto Congénito Gigante.
}

\author{
Hemangioma and Mixed Giant Congenital Lymphangioma.
}

\author{
Marlon Alexander Cerna Márquez* Larissa Maydelin Contreras Martínez*, Yolany Arely Casco Alvarado**, \\ Ramón Chinchilla, ${ }^{* * *}$ Roxana Martínez, ${ }^{* * * *}$ Héctor Rubén Caballero Castro*****
}

\section{RESUMEN}

Los linfangiomas son malformaciones hamartomatosas congénitas y en algunas ocasiones adquiridas que afectan la piel y el tejido subcutáneo, surgen cuando de forma localizada o generalizada se produce un cúmulo de conductos o quistes linfáticos. Los linfangiomas representan el $6 \%$ de los tumores benignos pueden aparecer en cualquier parte del cuerpo, el $75 \%$ se localizan en el cuello, la cabeza o las axilas. El 40-65\% están presentes al nacimiento. El tratamiento ideal del linfangioma depende del grado de infiltración, el tipo de tumor y la experiencia con el tratamiento. Se presenta caso clínico de recién nacido femenina, hija de madre de 21 años de edad, primigesta con embarazo de 34 1/7 semanas de gestación por FUM y sin antecedentes de relevancia, procedente de la aldea Monte la Virgen, Las Flores, Lempira. Nace en el Hospital Juan Manuel Gálvez, vía cesárea por fase latente prolongada. En la exploración física con múltiples masas de consistencia blanda con transiluminación positiva en cuello y tórax; extremidades asimétricas a expensas de neoformaciones de color rojo-violáceas en las superficies en asociación con hemangiomas. Se observa en piel lesiones eritematosas violáceas y tumorales tubero-cavernosas difusas múltiples, especialmente en extremidades superiores, tronco anterior derecho, extremidades inferiores y región vulvar. Se manejó en sala con Pipe-

\footnotetext{
* Residente de segundo año de Pediatría, Escuela Universitaria de Ciencias de la Salud. EUCS, UNAH-VS.

**Médico General. Autora independiente.

***Radiólogo del Instituto Hondureño de Seguridad Social, Hospital Regional del Norte. IHSS-HRN.

****Pediatra Hematooncologo del Hospital Mario Catarino Rivas. HMCR. *****Dermatologo Pediatra del IHSS-HRN.

Dirigir correspondencia a:marlon_alexander07@yahoo.com

Recibido: 03 de julio 2016 Aprobado: 27 de junio 2017
}

racilina/Tazobactam, Amikacina, Fluconazol, Inmunoglobulina, Propanolol, Prednisona y vincristina. La paciente falleció a los 8 días de vida.

\section{PALABRAS CLAVE}

Linfangioma, lesiones del sistema vascular, recién nacido.

\section{ABSTRACT}

Lymphangiomas are congenital and sometimes acquired Hamartomatousmalformations affecting the skin and subcutaneous tissue, arise when localized or generalized form an accumulation of lymph ducts or cysts occurs. Lymphangioma represents $6 \%$ of benign tumors can appear anywhere on the body, 75\% are located in the neck, head or armpits. $40-65 \%$ are present at birth. The ideal treatment of lymphangioma depends on the degree of infiltration, tumor type and treatment experience. We present a case report of a female newly born, first daughter of a 21-year-old mother with 34 1/7 weeks of gestation for FUM pregnancy and no history of relevance, from the village of Monte la Virgen, Las Flores, Lempira. Born in the Juan Manuel Galvez Hospital via caesarean section for prolonged latent phase. On physical examination with multiple masses of soft consistency with positive transillumination located in neck and chest; asymmetriclimbs at the expense of red-violet neoplasms in association with hemangiomas, erythematous lesions observed in skin, especially in upper extremities, right anterior trunk, lower limbs and vulvar area. It was handled with piperacillin / tazobactam, amikacin, Fluconazole, IV Immunoglobulin, propranolol, prednisone and vincristine. The patient died after 8 days of life. 


\section{KEYWORD}

Lymphangioma, vascular system injuries, Newborn.

\section{INTRODUCCIÓN}

Los linfangiomas son malformaciones hamartomatosas congénitas y en algunas ocasiones adquiridas tras acción traumática, infecciosa, iatrogénica o neoplásica, del sistema linfático, que afectan a la piel y al tejido subcutáneo, surgen cuando de forma localizada o generalizada se produce un acúmulo de conductos o quistes linfáticos. ${ }^{(1-3)}$

La patogénesis ha sido previamente estudiada. Se conoce que el drenaje de la linfa del embrión, cuya función es devolver a la corriente sanguínea sustancias proteicas y defender al organismo de las infecciones; se hace en el saco linfático yugular y de aquí la linfa drena por medio de un canal que se forma alrededor del día 40 posconcepcional en la vena yugular.

Un fallo en la formación del canal comunicante, un bloqueo de este o un aumento significativo de la presión venosa puede dar lugar a una retención de linfa en el sistema linfático, formando grandes quistes de retención alrededor de la región cervical que progresan a lo largo del cuerpo, con el desarrollo de un hidrops no inmune, provocando en algunos casos la muerte prematura del feto. Si luego se logra establecer un drenaje, la secuencia de acumulación de linfa se interrumpe y el exceso de linfa vuelve al sistema circulatorio fetal.(4)

Los linfangiomas representan el $6 \%$ de los tumores benignos, los casos malignosson raros y están asociados a radioterapia o a la aplicación de soluciones esclerosantes. Aunque pueden aparecer en cualquier parte del cuerpo, el $75 \%$ se localizan en el cuello, la cabeza o las axilas. Localizaciones menos habituales incluyen: el retroperitoneo (2\%), el mediastino (1\%) y área escrotal con aproximadamente 30 casos descritos en la literatura. ${ }^{(4-6)}$
El 40-65\% están presentes al nacimiento y un $80-90 \%$ se manifiestan antes de los 2 primeros años de vida, disminuyendo su aparición hacia los 16 años de edad. La incidencia de los linfangiomas congénitos gigantes es de 1/12.000 nacimientos y se observa con más frecuencia en el sexo masculino. ${ }^{(7-8)}$

El linfangioma se clasifica en capilar, cavernoso y quístico, pudiendo coexistir diferentes formas en un mismo linfangioma. La localización, estructura y consistencia de los tejidos circundantes pueden condicionar los diferentes tipos histológicos. $^{(2)}$ Usualmente son lesiones bien circunscritas y de un tamaño pequeño aunque en un menor número de casos pueden presentarse como lesiones gigantes. Sub clasificándose así por su tamaño microquísticos si es menor a $1 \mathrm{~cm}$ y macroquísticos si es mayor a $1 \mathrm{~cm}$. Existen linfangiomas mixtos de predominio macro o microquístico. ${ }^{(4)}$

Según su ubicación y tamaño se asocian a complicaciones en el manejo obstétrico y perinatal, que pueden ser consecuencia de: descompensación cardiovascular e hidrops fetal, obstrucción de la deglución fetal con polihidramnios secundario, prematurez, malformaciones asociadas, obstrucción del parto, dificultad en el establecimiento de la ventilación del neonato, y problemas de resección o terapia definitiva del tumor. ${ }^{(9)}$

Los datos histológicos más relevantes es la presencia de canales linfáticos que penetran y disecan las estructuras donde asienta el linfangioma. Esto confiere un comportamiento "agresivo» a estas lesiones benignas y es un dato muy importante a tener en cuenta en el momento de valorar bordes de resección quirúrgica y pronóstico de recidivas locales. ${ }^{(10)}$

Se publica este caso con el propósito de recordar que aunque se trata de una patología benigna, como parte del manejo, se debe evaluar el compromiso de estructuras vitales. 


\section{CASO CLÍNICO}

Se trata de recién nacido femenina, hija de madre de 21 años de edad, producto de la primera gestación y sin antecedentes de importancia, procedente de la Aldea Monte la Virgen, las Flores, Lempira. Se realizó 6 controles prenatales sin ninguna eventualidad $y$ varios ultrasonidos abdominales los cuales no reportaron ninguna malformación congénita. Madre es hospitalizada en el Hospital Dr. Juan Manuel Gálvez con embarazo de 34.1 semanas de gestación por FUM y Ruptura Prematura de Membranas de 5 horas de evolución por lo que le iniciaron conducción del trabajo de parto, no evolucionó satisfactoriamente por lo que le realizaron cesárea por fase latente prolongada.

Se obtuvo producto, único, vivo, femenina, cefálica, con APGAR 7 y 8 al primer y quinto minuto respectivamente, peso 2,962 gramos, 37 semanas de gestación por Capurro al cual se le brindaron los cuidados de rutina del recién nacido. En la exploración física se observaron múltiples masas de consistencia blanda en región de cuello y tórax; extremidades asimétricas a expensas de neoformaciones de color rojo-violáceas en las superficies en asociación con hemangiomas, se observaron en piel lesiones eritematosas violáceas y lesiones tumorales tubero-cavernosas difusas múltiples, especialmente en extremidades superiores, tronco anterior derecho, extremidades inferiores $y$ región vulvar (Ver figura No. 1). Los diagnósticos finales fueron: Hemangioma y Linfangioma Mixto Congénito Gigante, Sepsis Neonatal Tardía y Coagulación Intravascular Diseminada debido a que presentó leucocitosis, trombocitopenia, alteraciones en los tiempos de coagulación, Proteína $\mathrm{C}$ reactiva elevada y hemocultivo positivo para Klebsiella spp. Se manejó en sala con Piperacilina/Tazobactam, Amikacina, Inmunoglobulina para el manejo de la sepsis, Propanolol con el objetivo de provocar vasoconstricción y evitar neovascularización, Prednisona para evitar el crecimiento rápido del hemangioma e interrumpir el crecimiento y acelerar la involución y vincristina como inhibi- dor de la angiogénesis. La paciente falleció a los 8 días de vida.

Figura No. 1: A. Se observan tumoraciones gigantescas en tronco y extremidades de aspecto quístico y hemangiomatoso. B. Rayos X PA Tórax. Se observó marcado aumento de grosor y densidad de los tejidos blandos que involucra la región cervical.

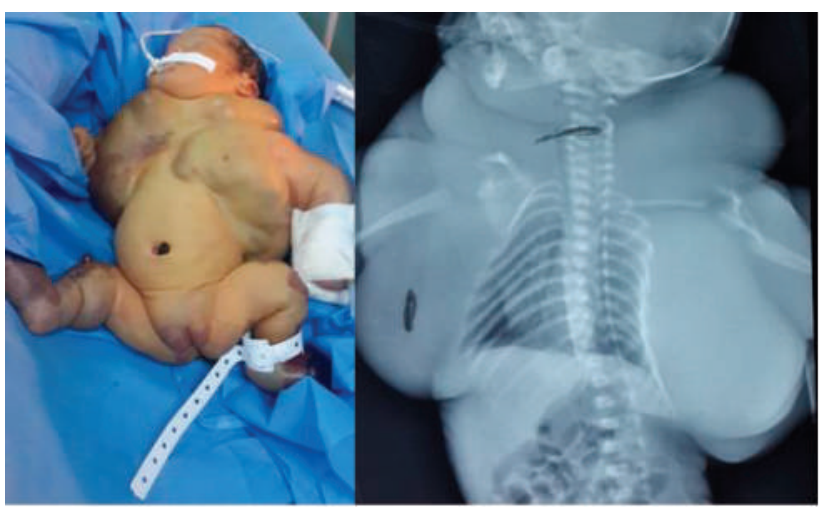

Fuente: Informe del Departamento de Radiología del HNMCR.

Fue referida al Hospital Nacional Dr. Mario Catarino Rivas (HNMCR) donde se le realizó Rayos $X$ PA de Tórax en el cual se observó un marcado aumento de grosor y densidad de los tejidos blandos que involucra la región cervical y axilar bilateral. No se logra identificar alteración pleuropulmonar. No se caracterizan las estructuras del mediastino por condición del paciente. En la Tomografía de Tórax se observó una alteración de la morfología torácica en relación con marcado aumento del tejido blando en región cervical y axilar bilateral con involucro de la pared anterior. En los tejidos antes descritos se constataron densidades heterogéneas sin definir zona de degeneración necrótica. Estructuras óseas conservadas. (Ver figura No. 1).

En la Tomografía de Cerebro (Ver figura No. 3) mostró ligera acentuación de cisuras y surcos cerebrales asociados a marcada disminución de la densidad de la sustancia blanca de ambos hemisferios en aparente relación por inmadurez cefálica. No se define hiperdensidad que sugiera sangrado intra o extra axial. Presencia de variante anatómica del septum pellucidum. 
En los cortes caudales se observó un aumento de grosor de los tejidos blandos en la región cervical por aparente proceso neoformativo a ese nivel.

\section{Figura No. 2: TAC Cerebral. La flecha señala acentuación de cisuras y surcos cerebrales.}

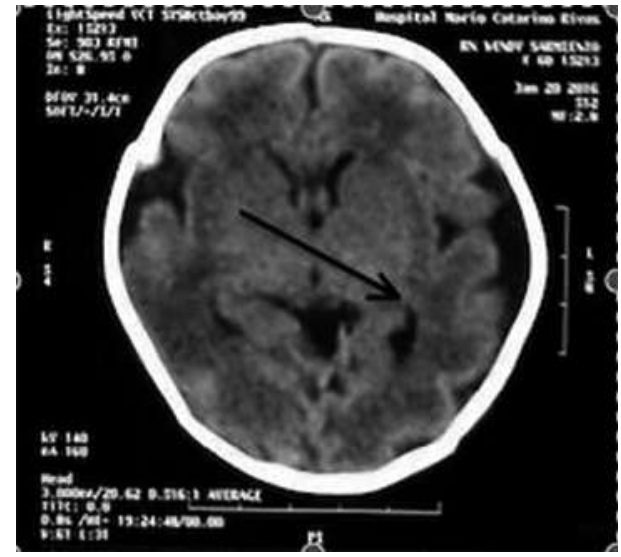

Fuente: Informe del Departamento de Radiología del HNMCR.

\section{DISCUSIÓN}

Los linfagiomas son hamartomas congénitos del sistema linfático.(1) Su etiopatogenia sigue siendo poco clara. ${ }^{(4)}$ Se consideran lesiones sin potencial maligno, sin embargo, suelen progresar, recidivar o presentar complicaciones como infección, obstrucción intestinal como necrosis, hemorragia intracavitaria o intraabdominal. ${ }^{(8)}$ Por lo general se tratan de lesiones quísticas, multiloculares, con contenido claro, quiloso o hemorrágico, pueden presentarse de manera única o múltiple como en el presente caso. ${ }^{(7)}$ Los datos histológicos más relevantes es la presencia de canales linfáticos que penetran y disecan las estructuras donde asienta el linfangioma. Esto confiere un comportamiento «agresivo» a estas lesiones benignas y es un dato muy importante a tener en cuenta en el momento de valorar bordes de resección quirúrgica y pronóstico de recidivas locales ${ }^{(10-11)}$ El diagnóstico de las malformaciones linfáticas se puede hacer de manera prenatal, por ultrasonografía al término del primer trimestre de gestación; sin embargo, puesto que la mayoría de las lesiones se detectan después del nacimiento, la radiografía simple puede ser la primera prueba diagnóstica que documente posibles anomalías asociadas dependiendo de la localización de la lesión se puede observar aumento del grosor y densidad de los tejidos blandos. $^{(9)}$

La ecografía, principalmente el Eco Doppler, es muy útil para definir la extensión y la naturaleza de la malformación cuando ésta es superficial; es menos útil para demostrar lesiones extensas en el cuello, mediastino o retroperitoneo, en cuyo caso se recurre a la tomografía axial computarizada o a la resonancia magnética tiene muy buena resolución en estructuras que afectan cráneo, cuello, tronco, mediastino y extremidades, además nos da mayor definición, extensión de la lesión y visión de las estructuras vecinas. ${ }^{(10)}$ Tanto la tomografía como la resonancia pueden ser de utilidad para las lesiones abdominales, retroperitoneales o que afectan órganos sólidos internos. ${ }^{(9-10)} \mathrm{Al}$ paciente se le realizaron Rayos X PA de Tórax y TAC Full Body Scan en donde se lograron observar la extensión y profundidad de los linfangiomas.

El tratamiento de elección del linfangioma es la resección quirúrgica completa o bien mediante esclerosis de la lesión por acción de agentes esclerosantes. Entre ellos destaca el OK-432, también llamado Picibanil, que es un compuesto liofilizado de baja virulencia, sustraído de la bacteria del estreptococos pyogenes del grupo A. Está contraindicado su uso en pacientes alérgicos a b-lactámicos por riesgo de anafilaxia. ${ }^{(10,12)}$ Algunos autores utilizan la terapia con láser para paliar linfangiomas en localizaciones peculiares, y para el control de hemorragias. Existe la posibilidad de recidiva local, hay una evidencia del $10-18 \%$ de recurrencias. ${ }^{(11,12)}$

Castañón y cols. aportan un nuevo tratamiento para las recidivas de linfangiomas con inyección intraquística de adhesivo de fibrina, tras aspiración y evacuación del contenido líquido del quiste. ${ }^{(10)}$ 
El tratamiento ideal del linfangioma depende del grado de infiltración, el tipo de tumor y la experiencia con el tratamiento. El linfangioma gigante, o macroquístico, siempre tiene mejor pronóstico que el microquístico en cualquiera de los tratamientos, y sobre todo si su componente es quístico y no cavernoso o mixto. ${ }^{(9)} \mathrm{La}$ paciente fue manejada con cobertura antibiótica (Piperacilina/Tazobactam, Amikacina y Fluconazol) e inmunoglobulina por su proceso de sepsis, además se le suministro vincristina y propanolol por su hemangioma, sin mostrar efectividad en el tratamiento; no se usó OK-432 y otros agentes esclerosantes ya que no hay en existencia en la institución, tampoco se realizó la resección quirúrgica por el compromiso de estructuras vitales y altas posibilidades de sangrado masivo, manejándose conservadoramente. Desafortunadamente, la paciente falleció a los 8 días de vida por sepsis neonatal y coagulación intravascular diseminada.

El caso presentado, se trata de un padecimiento raro y con pronóstico ominoso. No existe en la literatura una casuística amplia ni con un seguimiento a largo plazo de los pacientes, la mayor parte de los artículos revisados para este reporte son informes de casos. Se propone que la conducta ideal deberá individualizarse, según hallazgos y condiciones clínicas del paciente la experiencia del cirujano y las condiciones logísticas del medio hospitalario. El tratamiento debe ser multidisciplinario para aumentar la supervivencia de los pacientes.

\section{BIBLIOGRAFÍA}

1. García Eco MD, Jiménez Fernández I, Carrera Trugeda MS, Gonzales Sandoval F. Hemangiomas y malformaciones vasculares. ¿ Qué se puede hacer?. Bol pediatr. [Internet] 2001 [Citado, 16 Enero 2016]; 50(41):137-143. Disponible en: http://www. sccalp.org/boletin/176/BolPediatr2001 _41_137-143.pdf.

2. Ortiz Rodríguez JA, Gonzáles Urzola ES, Ortiz Alcoser VA, Baquero Rodrigo P. Linfangioma fetal: reporte de casos en Bogotá, Colombia. Mév. Med. UIS. [Internet]2012 [Citado, 13 enero 2016];25(2):149-54. Disponible en: http://revistas.uis.edu.co/ index.php/revistamedicasuis/article/view/ $3165 / 3900$.

3. CruzFontirroche PR, Dalmau GonzálezL, Riol BarroetabeñaY, Mejías Araujo M. Linfangioma quístico abdominal. A propósito de dos casos pediátricos. Mediciego. [internet] 2010 [Citado, 14 Ener2016]; 16(1):154-63. Disponible en: http://bvs.sld. cu/revistas/mciego/vol16_supl1_10/casos/ t-14.html.

4. Beltrán MA, Barría C, Pujado B, Oliva J, Contreras MA, Wilson CS, et al . Linfangioma esplénico gigante: caso clínico. Rev. méd. Chile [Internet]. 2009 [Citado 05 abril 2016];137(12):1597-1601. Disponible en: http://www.scielo.cl/scielo.php?script= sci_arttext\&pid=S0034-98872009001200 008\&lng=es.

5. González Torres S, Navarrete G, Llanos Villagómez E. Hemangiolinfangioma. Rev Cent Dermatol Pascua. [Internet] 2008 [Citado 02 febrero 2016];17(1):26-29. Disponible en: http://www.medigraphic.com/pdfs/der ma/cd-2008/cd081f.pdf.

6. Díaz Avilés $P$, Del campo MN, Archundia Hernández $E$, Rabago Rodriguez $M$, Reyes AP, Iriarte Tinajero M. Diagnóstico y manejo perinatal del linfangioma gigante de cuello. Acta Pediatr Esp. [Internet] 2007 [Citado 
02 febrero 2016]; 41 (65);32-35. Disponible en: http://webcache.googleusercontent. com/search?q=cache:-Shj9MbSX30J:www actapediatrica.com/index.php/secciones/ notas-clinicas/download/431_1a87 bc926342e040b55a523f53d5e9eb+\&cd= $1 \& \mathrm{hl}=\mathrm{es} \& \mathrm{ct}=\mathrm{clnk} \& \mathrm{gl}=\mathrm{hn}$.

7. Guerra, F, Jiménez Rodríguez $P$, García Rubilar M, Izaguirre Isla A, Sosa IA, Bardales Guerra J, et al. Linfangioma cervical cavernoso en un gemelo: análisis crítico del diagnóstico y manejo perinatal. Revista chilena de obstetricia y ginecología. [Internet] 2007 [Citado 05 Abril 2016]; 32(72): 335-341. Disponible en: http://www.scielo. cl/scielo.php?script=sci_arttext $\&$ pid= S0717-75262007000500010.

8. Aranguez MontoyaG. Aspectos clínicos y morfológicos de los linfangiomas infantiles: Revisión de 145 casos. An Esp Pediatr. [Internet] 1996 [Citado 05 abril 2016]; 25(4): 25-28. Disponible en: https://www. google.hn/url?sa =t\&rct=j\&q=\&esrc=s\&sou $r c e=$ web $\& c d=2 \& c a d=r j a \& u a c t=8 \& v e d=0 a$ hUKEwjEjZng9vHOAhWKmx4KbAkCwQ QFggfMAE\&url=http\%3A\%2F\%2Fwww.elsevier.es\%2Fes-revista-cirugia-espano la-36-pdf-S0009739X01717277-S300\&us g=AFQjCNGnOY93KoM8HrGro3xJXHTNQZ GJlw\&bvm=bv.131783435,d. dmo.

9. Navarro Mancía A, Romero Sagastume J, C Peña Cabús. Linfangioma quístico axilar gigante del adulto: características de presentación y abordaje quirúrgico. Cir. plást. iberolatinoam. [Internet]. 2015 [Citado 05 abril 2016];41(3):315-320. Disponible en: http://scielo.isciii.es/scielo.php?script= sci_arttext\&pid=S0376-789220150003000 $12 \&$ Ing=es. http://dx.doi.org/10.4321/S03 76-78922015000300012.

10. Huerta-Saenz MA, Castillo Mejía I, Montoya Fuerez E, Reyes JC. Linfangioma cervical fetal: diagnóstico prenatal y resultado perinatal. Rev. Peru. Ginecol. Obstet. [Internet] 2012 [Citado 02 febrero 2016]; 58(4): 347-350. Disponible en: <http:// www.scielo.org.pe/scielo.php?script $=$ sci_arttext\&pi$d=S 2304-51322012000400013 \&$ Ing $=e s \& n$ $\mathrm{rm}=\mathrm{iso}>$.

11. Mengual Ballester M, Montoya Chinchilla R, Guillén Paredes MP, Aguayo Albasini JL. Graves consecuencias del tratamiento mediante esclerosis con ácido acético en un caso de linfangioma quístico retroperitoneal. Actas Urol Esp. [Internet] 2010 [Citado 05 abril 2016]; 34(10): 913-915. Disponible en: http://scielo.isciii.es/scielo.php?script=sci_arttext\&pid=S0210-48 062010001000023\&lng=es.

12. Weber Sánchez A, Maya GD, Vega Rivera F, Bravo Torreblanca C, Martínez DG, Carbo Romano R. Resección laparoscópica de linfangioma cavernoso retroperitoneal residual. Cir. gen [Internet] 2012 [Citado 05 abril 2016]; 34(3): 206-212. Disponible en: http://www.scielo.org.mx/scielo.php?scri pt=sci_arttext\&pid=S1405-009920120003 $00011 \&$ lng $=$ es. 